\title{
The introduction of spin to an existing flexible shot model within a gun dynamics simulation
}

\author{
David N. Bulman \\ Danby Engineering Ltd., High Gill House, Low Row, \\ Richmond, North Yorkshire, DL11 6NE, UK \\ Tel.: +44 1748 886345; Fax: +441748 886014
}

\begin{abstract}
The paper describes the enhancement of an existing gun dynamics simulation package, to introduce spin to the flexible shot model. The original one or two piece flexible shot model was based on finite element beam theory, and therefore spin needed to take into account the moments of inertia at each node. Each node is therefore treated as an independently spinning body, each with its own spinning axes which can be related to the next node so that stiffness forces between them can be calculated. The stiffness coefficients are recalculated as deformation takes place, which is particularly important with long rod penetrators because of the high accelerations involved which induce buckling moments in the rod as it bends. The relationship between the nodes and the stiffness coefficients is maintained by updating the direction cosines for each node, plus a set for the overall beam.

The final model has been validated against known rigid model cases, by increasing stiffness until flexing is reduced. Comparisons have then been made with standard whirling cases.
\end{abstract}

\section{Introduction}

The modelling of barrel and shot motion during firing is now an accepted part of gun design procedure. Analysis has been carried out on guns with bore sizes ranging from $12.5 \mathrm{~mm}$ to $155 \mathrm{~mm}$, and in some cases it is known that the models have been used on a bore size as small as $6 \mathrm{~mm}$ [1]. Comprehensive simulations now include flexible and recoiling barrels, effects of bore wear, non-linear supports, flexible cradles, rigid or flexible shots, and the supporting vehicle [1-3]. The rigid shot models have usually included spin and the mathematics is well understood. Originally the flexible shot models were specifically designed to analyse the long rod penetrators of $120 \mathrm{~mm}$ APFSDS rounds. In this case spin is of less importance as in many cases they are fired from smooth bore barrels, or have slipping driving bands which keep spin to a controlled minimum.

The first non-spinning flexible shot models were based on non-spinning flexible beams $[4,5]$. They have been shown to give very good results with the smooth bore guns for which they were initially designed. However, even with slipping driving bands, the spin induced in a shot can be considerable and the use of nonspinning flexible shot models for these cases is not acceptable. Furthermore, gun dynamics modelling is being used more extensively with smaller calibres which include rifling, and with an increased requirement to model long rod penetrator type shots. The ability to simulate spinning flexible shots is therefore essential in a comprehensive gun dynamics simulation.

This paper describes the enhancement of an existing gun dynamics simulation package, SIMBAD [2], to introduce spin to the flexible shot model. The original one or two piece flexible shot model was based on finite element beam theory [5]. This type of model was used because of its simplicity, and its ability to produce good results with relatively few elements. It keeps simulation times short, and enables parameter studies to be made with ease. More complex models, using 'brick' elements [6,7], can offer the possibility of providing a deeper understanding of the deformation of the shot, but require a much greater time to develop a specific model, and the run times can be very long which makes their use in parameter studies unlikely.

Most dynamic simulations which use finite element methods assume the inertia forces for a particular freedom at each node to be equal to the acceleration multiplied by the mass. This also applies to nodes with moments of inertia where the inertia torques are assumed to be equal to the angular acceleration multiplied by the moment of inertia. For elements which are only described by point masses at each node (normally brick elements with 3 degrees of freedom at each node) this assumption is valid for any general motion. For elements which are described by point masses plus moments of inertia at each node, this assumption is valid for any general motion provided that the angular veloc- 
ities are small enough so that 'gyroscopic' effects can be neglected. This applies to beam models which have 6 degrees of freedom at each node relating to the three translations and three rotations.

It is for this reason that the simple dynamic analysis used with beams can not be used for the analysis of spinning flexible shots. Each node needs to be treated as an independently spinning body, each with its own spinning axes. The 'gyroscopic' inertia torques then need to be included in the analysis, and the motion of one node related to the next node so that stiffness forces between them can be calculated. This requires the stiffness coefficients to be known, and these need to be recalculated as deformation takes place. This is particularly important with long rod penetrators because of the high accelerations involved which induce buckling moments in the rod as it bends [5]. (Note: This is important with all types of elements.)

\section{Basic theory}

The existing model from which the final model was constructed is based on standard finite element type theory. The shot is divided into a number of beam elements, and a stiffness matrix is produced so that:

$$
[F]=\left[F_{s}\right]
$$

where $\left[F_{s}\right]=[K][\delta]$ and may be known as the stiffness force vector.

This is the normal equation used in the finite element analysis of structures where the stiffness matrix $[K]$ relates the displacements $[\delta]$ at the nodes to the applied forces $[F]$ at those nodes. The displacements include the longitudinal and transverse displacements, and also the angular displacements about three fixed orthogonal axes, normally known as the global axes, $O x_{g} y_{g} z_{g}$. It will be seen later that $\left[F_{s}\right]$ requires special treatment when the shot is spinning.

In a dynamic situation $[F]$ is a function of time, and will be composed of externally applied forces plus inertia and damping forces. The equation will then hold true at any instant in time. Hence, let:

$$
[F]=\left[F_{t}\right]-[M][\ddot{\delta}]-[C][\dot{\delta}]
$$

where $\left[F_{t}\right]$ are the applied external forces which may be time dependent,

$[M]$ are the equivalent masses and inertias of the shot at the nodes, and in this case is a column vector

$[C]$ are the viscous damping coefficients at the nodes, a column vector $[\dot{\delta}]$ are the global velocities, and $[\ddot{\delta}]$ are the global accelerations.

Replacing $[F]$ by $\left[F_{s}\right]$ from Eq. (1), the 'standard' form of Eq. (2) can be written:

$$
[M][\ddot{\delta}]+[C][\dot{\delta}]+\left[F_{s}\right]=\left[F_{t}\right]
$$

Equation (3) represents a set of simultaneous second order differential equations. The formation of the relevant matrixes $[M]$ and $[C]$ is already well documented, and it is now a relatively simple matter to solve the equations for any particular combination of applied forces $\left[F_{t}\right]$. For a conventional gun, the forces consist of the propellant gas pressure forces (normally on the base), and the reactions between the shot and the bore. For an electromagnetic gun, the pressure forces may be replaced by a distributed force along the shot which accelerates it along the bore. The reactions with the bore can be obtained by assuming a non-linear elastic interface, with or without clearance $[1,2,8]$. The actual solution is made by rearranging the equations so that

$$
[\ddot{\delta}]=[M]^{-1}\left[F_{\text {all }}\right]
$$

where $\left[F_{\text {all }}\right]=\left[F_{t}\right]-[C]\left[\dot{\delta}-\left[F_{s}\right]\right.$

A modified Runge-Kutta routine is then used to solve Eq. (4).

These equations are satisfactory for a non-spinning shot, provided that the stiffness coefficients are recalculated during the simulation [5]. When the shot is spinning such that the gyroscopic torques are significant the term $[M][\ddot{\delta}]$ is no longer valid for the rotational degrees of freedom. Also the simple form of the stiffness force vector, $\left[F_{s}\right]=[K][\delta]$, will no longer be valid, and must now take into account the rotation of the shot.

First consider the changes due to the effect of the gyroscopic torques. For a single node, the linear motion in the global directions is valid and can remain, but the 'inertia' torques must be replaced by Euler's Dynamical Equations. In this case

$$
\begin{aligned}
& Q_{x}=I_{x x} \dot{\omega}_{x}-\left(I_{y y}-I_{z z}\right) \omega_{y} \omega_{z} \\
& Q_{y}=I_{y y} \dot{\omega}_{y}-\left(I_{z z}-I_{x x}\right) \omega_{x} \omega_{z} \\
& Q_{z}=I_{z z} \dot{\omega}_{z}-\left(I_{x x}-I_{y y}\right) \omega_{x} \omega_{y}
\end{aligned}
$$

where $Q_{x}, Q_{y}, Q_{z}$ are the torques on each axis (damping, stiffness and applied),

$I_{x x}, I_{y y}, I_{z z}$ are the principal moments of inertia about each axis,

$\dot{\omega}_{x}, \dot{\omega}_{y}, \dot{\omega}_{z}$ are angular accelerations about each axis, and $\omega_{x}, \omega_{y}, \omega_{z}$ are angular velocities about each axis. 
All the terms in these equations refer to the inertia torques about the principal axes of the nodal mass. These will be referred to as the local axes, $O x_{l} y_{l} z_{l}$, for the node, which are fixed to the node and move with the node. A transfer matrix, $\left[T_{L}\right]$, containing the direction cosines of the local axes, must therefore be set up to relate the local axes of the node to the global axes. Let

$$
[\mathrm{GLOBAL}]=\left[T_{L}\right] *[\mathrm{LOCAL}]
$$

Therefore, referring to Eqs (4) and (5), for the rotational freedoms of a single node we can write:

$$
\begin{aligned}
{[\dot{\omega}]=} & {\left[M_{s}\right]^{-1} *\left[[ T _ { L } ] ^ { - 1 } \left[\left[F_{s t}\right]-\left[C_{s}\right][\dot{\theta}]\right.\right.} \\
& \left.\left.-\left[F_{s s}\right]\right]+[R]\right]
\end{aligned}
$$

where $[\dot{\omega}]$ contains $\dot{\omega}_{x}, \dot{\omega}_{y}, \dot{\omega}_{z}$,

$\left[M_{s}\right]$ contains $I_{x x}, I_{y y}$ and $I_{z z}$.

$\left[F_{s t}\right]$ contains the associated terms from $\left[F_{t}\right]$,

$\left[C_{s}\right]$ contains the associated terms from $[C]$,

$[\dot{\theta}]$ contains the associated terms from $[\dot{\delta}]$,

$\left[F_{s s}\right]$ contains the associated terms from $\left[F_{s}\right]$,

and $[R]$ contains terms $\left(I_{y y}-I_{z z}\right) \omega_{y} \omega_{z},\left(I_{z z}-\right.$ $\left.I_{x x}\right) \omega_{x} \omega_{z},\left(I_{x x}-I_{y y}\right) \omega_{x} \omega_{y}$.

Equation (7) can be written as

$$
[\dot{\omega}]=\left[M_{s}\right]^{-1} *\left[F_{r}\right]
$$

If we take $\left[F_{\text {all }}\right]$ from Eq. (4) and replace the components concerned with the rotational freedoms with the terms from $\left[F_{r}\right]$ for each node, we can write for the complete shot:

$$
\left[\ddot{\delta}_{\mathrm{LG}}\right]=[M]^{-1}\left[F_{\text {allLG }}\right]
$$

where $\left[\ddot{\delta}_{\mathrm{LG}}\right]$ contains the Local rotations and Global linear motion,

and $\left[F_{\text {allLG }}\right]$ contains the Local moments and Global linear forces.

Provided that $\left[F_{s}\right]$ is calculated as shown below, the solution of this can now be made in the same way as Eq. (4), and will give the global linear velocities and displacements, plus the local angular velocities and displacements. For each step in the solution, the step increments in angular displacements about the local axes can be used to update the transfer matrix $\left[T_{L}\right]$.

It is still necessary to know the global angular velocities and displacements, both to appreciate the motion of the shot, and to calculate some of the terms required in $\left[F_{t}\right]$. For each node, the global angular velocities can be derived directly by using Eq. (6). It is not possible to use the transfer matrix to convert angular displacements, but for the relatively small angular displacements expected about $y_{l}$ and $z_{l}$ we can show that the global angular displacement about $x_{g}$ is the same as the local angular displacement about $x_{l}$. Also for the angular displacement about $y_{g}$ and $z_{g}$ we can use the projection of the local $x_{l}$ axis onto the global $x_{g} z_{g}$ and $x_{g} y_{g}$ planes respectively. This gives:

angular displacement about global

$y=-T_{L}(3,1)$

angular displacement about global

$z=T_{L}(2,1)$

Hence we can update $[\delta]$ and $[\dot{\delta}]$, which are then used to obtain the relative displacements and velocities between the shot and the barrel, and hence the contact forces at the shot bands.

We must now consider the recalculation of the stiffness coefficients during the solution $[2,5]$ and the calculation of the stiffness force vector, $\left[F_{s}\right]$, given in Eq. (1). It may be noted that the term $[K][\delta]$ referred to the global directions, but because the shot is spinning the stiffness terms in $[K]$ refer to the spinning directions, and $[K]$ is not valid to be used with $[\delta]$ which is in global directions.

We therefore introduce another set of axes, $O x_{s} y_{s} z_{s}$, initially coincident with the global axes, but which have angular motion about the global $x_{g}$ axis which is equal to the spin angle of the node at the driving band of the shot. These therefore spin with the shot, and the shot then has small displacements relative to these axes. These displacements need to be found so that the new stiffness coefficients and the stiffness forces can be calculated in these directions. We introduce another transfer matrix so that:

$$
[\mathrm{SPIN} A X E S]=\left[T_{S}\right] *[\text { GLOBAL }]
$$

The linear displacements at a node in the spin axes directions $x_{s}, y_{s}$ and $z_{s}$ can be given directly by the above equation. For small angular displacements about local $y_{l}$ and $z_{l}$, we can show that the angular displacement about the spin axis $x_{s}$ is the same as the local angular displacement about $x_{l}$ minus the angular displacement of the spin axes. For the angular displacement of a node about spin axes $y_{s}$ and $z_{s}$ we can use the projection of the local $x_{l}$ axis onto the $x_{s} z_{s}$ and $x_{s} y_{s}$ planes of the spin axes respectively. It can be shown that this is given by:

$$
\begin{aligned}
& \text { angular displacement about spin axes } y= \\
& -\left(T_{S(3,2)} \cdot T_{L(2,1)}+T_{S(3,3)} \cdot T_{L(3,1)}\right)
\end{aligned}
$$




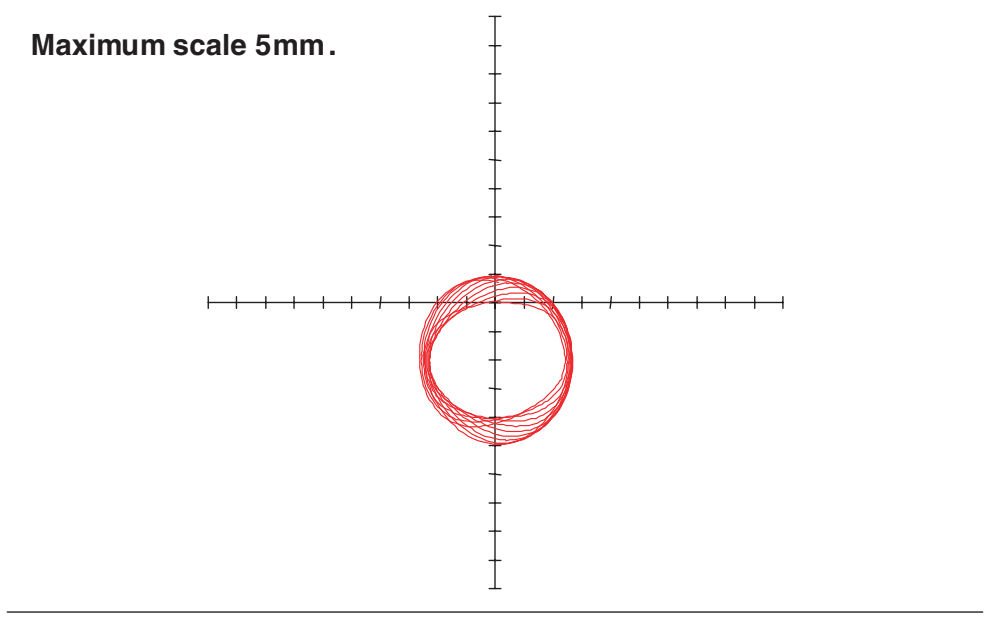

Z-Y motion of the central node

Fig. 1. Spin speed $10 \mathrm{~Hz}$.

angular displacement about spin axes $z=$

$T_{S(2,2)} \cdot T_{L(2,1)}+T_{S(2,3)} \cdot T_{L(3,1)}$

To calculate the new stiffness coefficients during the simulation, the $y_{s}$ and $z_{s}$ displacements calculated above are added to the original positions of the nodes at the start of the simulation. The stiffness coefficients are then calculated in the usual way.

The stiffness forces are calculated by taking the new stiffness matrix and multiplying it by the calculated linear and angular displacements in the spinning axes directions. The transfer matrix $\left[T_{S}\right]$ is then used to convert these stiffness forces to the global directions to give the term $\left[F_{s}\right]$ which is required in Eqs (4) and (7).

In practice, the actual model is specifically designed for use with a one or two piece shot. The two piece shot involves a separate sabot and penetrator, and in this case each part is simulated individually with an interaction between the two parts. This interaction assumes a circular cross-section of the penetrator and sabot at the interface, and is based on the resultant relative displacement between the two parts [8]. This was unchanged between the non-spinning and spinning models.

\section{Validation}

It is very difficult to obtain good experimental data to validate the complex model of a spinning flexible shot. The only satisfactory method would be to measure the flexing of the shot as it travelled down the bore. There are great practical difficulties with this, although some work has been done for non-spinning flexible shots [6, 7,9] and methods for measuring pitch and yaw for rigid shots have been developed [10,11]. However, at the time of writing no experimental data is known by the author for the complete motion of a spinning flexible shot as it travels down the barrel. A different approach was therefore taken.

The first part of the validation was to remove the pressure and band forces from the shot, and then support the shot at either end in pin-joints. This was done in the program through the 'User Defined Routines' [2]. A small bend was placed in the shot which was then spun at various speeds to encourage a whirling condition. In this case the shot was a simple thin rod, for which the natural whirling frequency could be calculated.

For each speed, the transverse displacement of the centre node was recorded and the simulation continued until steady state conditions were achieved. The amplitude of the displacement was plotted against speed, and the result compared with the theoretical whirling frequency. This was repeated for a two piece shot, in which the composite of the two pieces was equivalent to the single rod, and the interface was made very stiff. In order to keep run times to a minimum, only 10 elements were used for each part, and the thickness and the length of the rod was such as to keep the natural frequency relatively low.

The results showed that the maximum amplitude occurred at the expected natural whirling frequency. Figures 1 to 3 show the motion of the centre node in a plane at right angles to the spin axis from the start of the simulation up to a time of 1 second. It may be noted that the zero point on the plot is the starting point of 


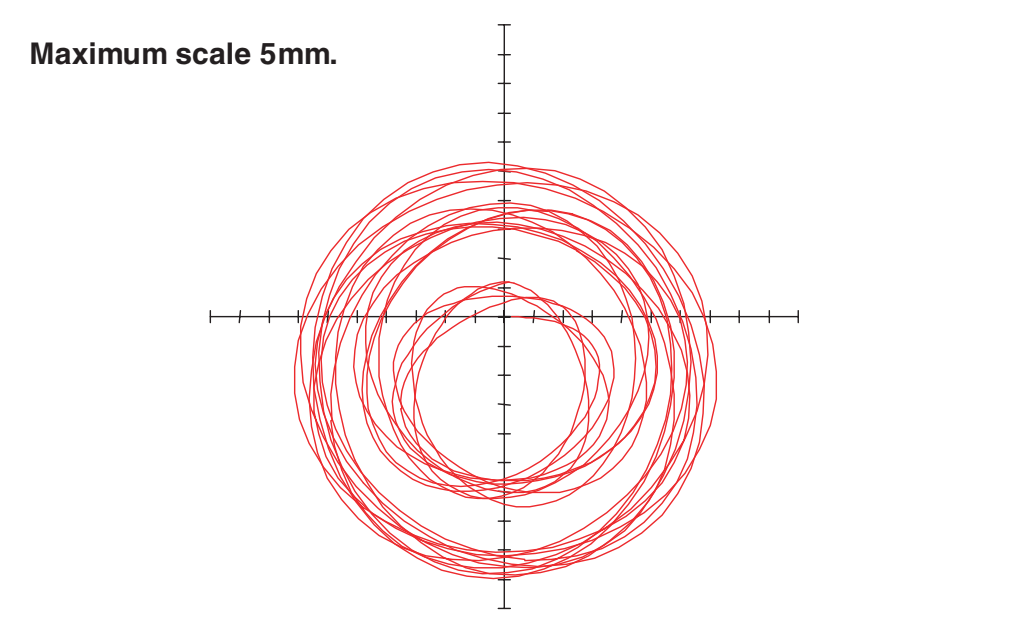

\section{Z-Y motion of the central node}

Fig. 2. Spin speed $20 \mathrm{~Hz}$.

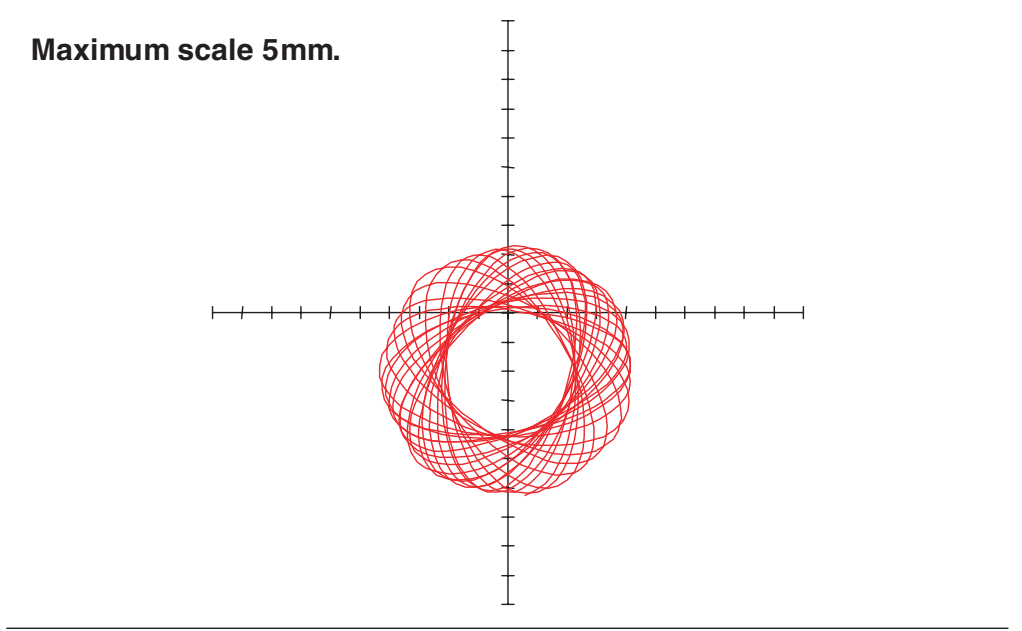

\section{Z-Y motion of the central node}

Fig. 3. Spin speed $40 \mathrm{~Hz}$.

the node, which was $1 \mathrm{~mm}$ above the spin axis. It can clearly be seen that at the natural frequency of $20 \mathrm{~Hz}$ the amplitude was increasing rapidly. The reason why the motion is not symmetrical is that at the start of the simulation the transverse velocity of the node was given a value equal to the spin speed multiplied by the offset. This starting condition is similar to what would occur to a node if the rod was already spinning, but it does not include the centripetal acceleration and the corresponding deflection of the rod. However this starting condition enabled the final steady condition to be reached quicker than if the nodes had initially been stationary.
The result gave confidence in the ability of the program to simulate a spinning flexible rod, but more evidence was required to confirm that the gyroscopic torques were included correctly, and that the complex pressure forces and shot band forces were being simulated. This was done by comparison with a well validated spinning rigid shot.

A model of an actual shot was composed using both the rigid shot simulation and a two piece flexible shot simulation. Each shot therefore had the same total mass, inertias, position of the centre of gravity, and contact geometry with the bore. Each shot had the same band geometry and elastic properties, including 


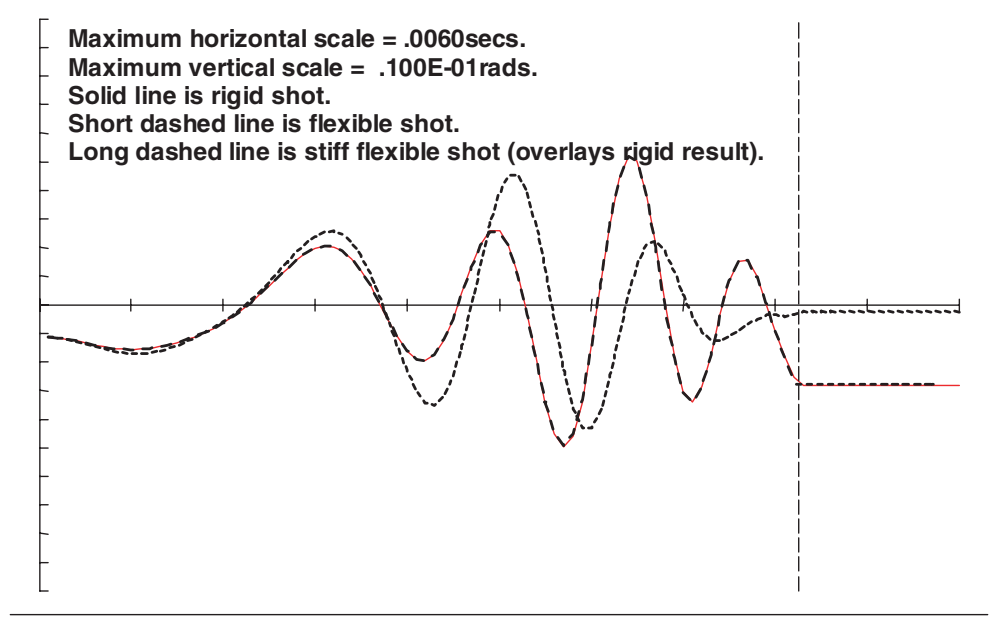

The three cases.

Rigid shot, flexible shot and stiff flexible shot.

Fig. 4. Shot pitch angle.

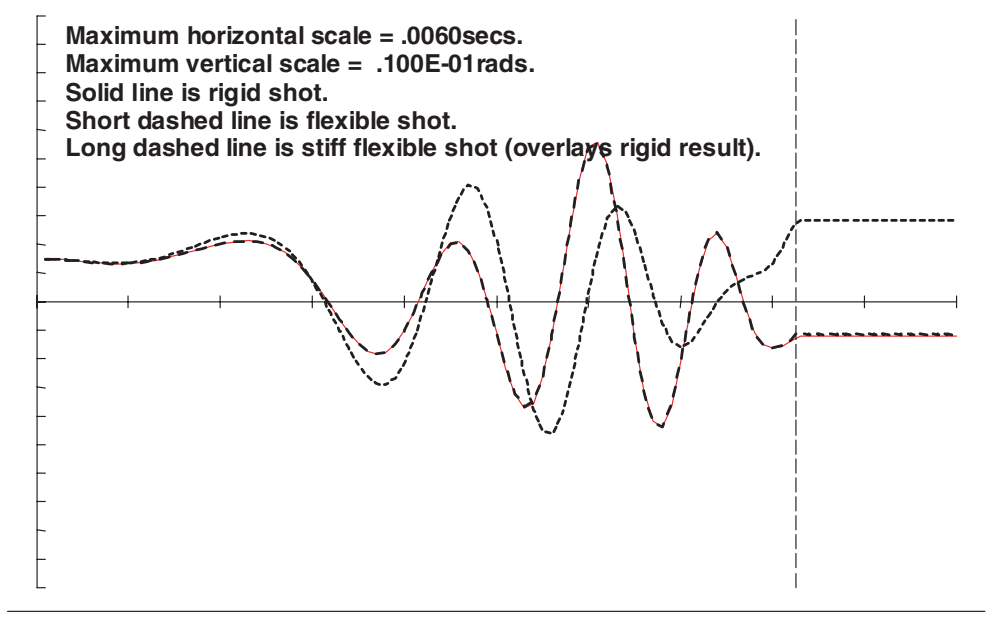

The three cases.

Rigid shot, flexible shot and stiff flexible shot.

Fig. 5. Shot yaw angle.

clearance on the front band. They were subjected to the same pressure forces, and each included a slightly angled driving band. The longitudinal principal axis of the shot was also offset slightly from the centre of the driving and centring bands. In practice, if no flexing occurs, these two models should give the same results. Simulations were therefore made, and repeated with the modulus of elasticity increased progressively to a factor of 100. Various parts of the results were examined, including the bending of the flexible shot.

Figures 4 and 5 each show three results for the shot pitch and yaw. The first is for the rigid shot, the second is for the flexible shot with the modulus of elasticity increased by a factor 100 , and the third is for the flexible shot with the correct modulus of elasticity. The first and second results overlay each other. This showed clearly that, on the flexible shot, the additional gyroscopic effects, plus the band forces and the pressure forces, were being simulated correctly. It should also be noted that to achieve such a good match the stiffness coefficients were recalculated at every time step.

As a further point of validation, the actual bend in 


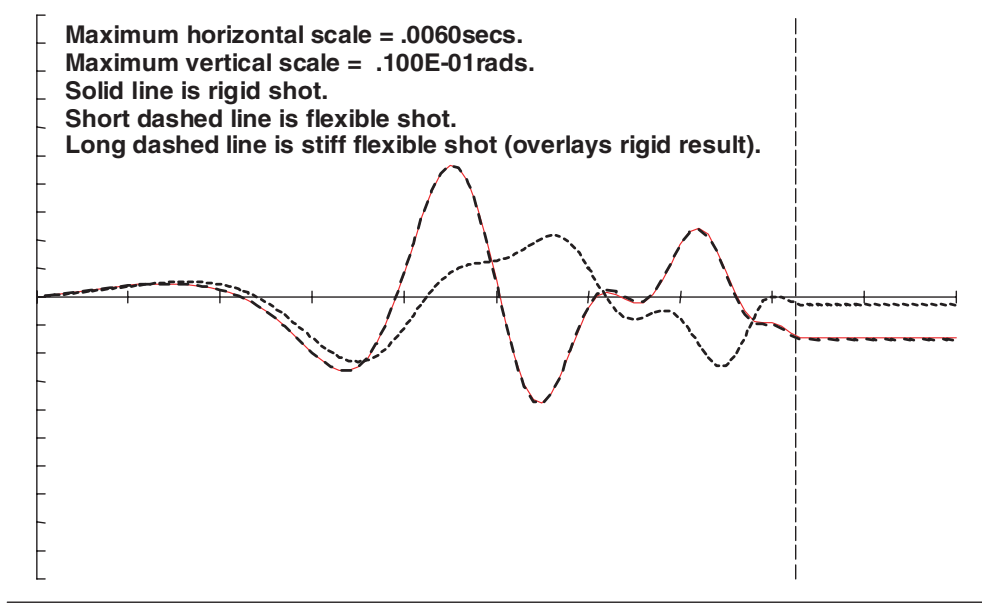

The three cases.

Rigid shot, flexible shot and stiff flexible shot.

Fig. 6. Shot vertical bounce velocity.

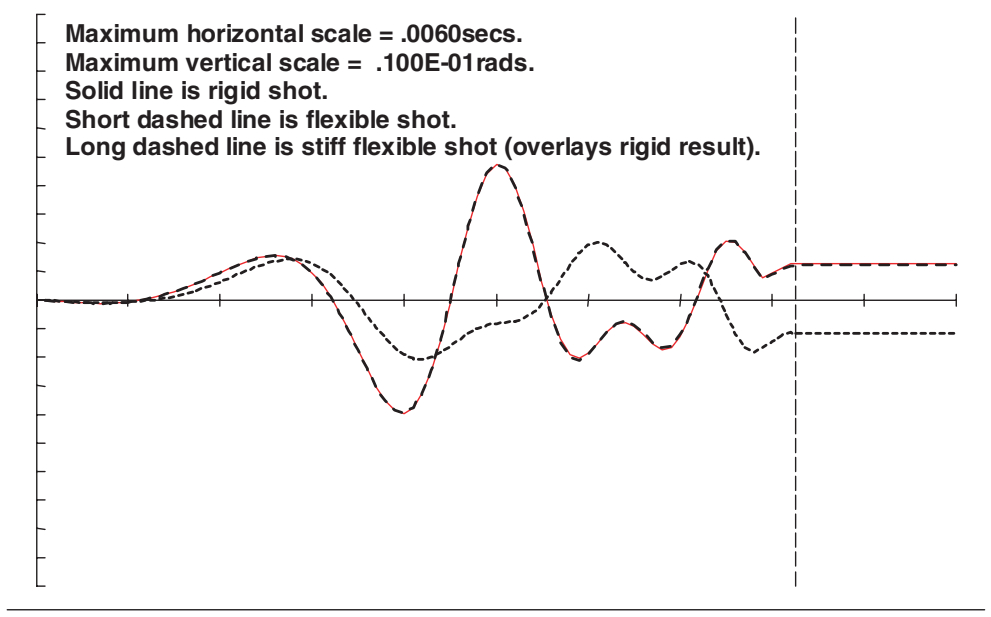

The three cases.

Rigid shot, flexible shot and stiff flexible shot.

Fig. 7. Shot horizontal bounce velocity.

the shot was about $1 \mathrm{~mm}$ at shot exit. This compares with measured values of similar shots taken from photographs just after shot exit.

\section{The significance of spin and flexibility}

In previous work with long thin spinning projectiles, such a APFSDS, the shot has been simulated by either a non-spinning flexible model, or a rigid spinning model. The importance of including the dynamics of a spinning projectile is already well known, but it can now be seen that the omission of the bending of the shot can give significant errors in the final predictions of shot launch.

In order to compare results between the rigid and flexible shot simulations, definitions for the flexible shot of bounce and bounce velocity in both $y_{g}$ and $z_{g}$ directions, pitch and yaw angles, and pitch and yaw velocities, need to be considered.

The shot bounce in each direction, at each point in time, is defined as the position of the centre of gravity of the shot taking into account the deflected shape of the round. This is found by taking the moments of the 
mass at each node about the $y_{g}$ or $z_{g}$ axes. They are summed, and the result divided by the total shot mass.

The bounce velocities are found by calculating the linear momentum of the mass at each node in the directions $y_{g}$ and $z_{g}$. These are summed for each direction, and the results divided by the total shot mass to give the bounce velocities of the complete shot.

The shot pitch and yaw are calculated by making a straight line least squares fit of the nodal positions. The angle of this line then gives the pitch and yaw values.

The pitch and yaw velocities are found by calculating the total angular momentum about the $z_{g}$ and $y_{g}$ axes respectively. These are then divided by the shot inertias about the relevant axes.

Using the shot described above, which was simulated with the flexible and rigid models, Figs 4 and 5 show the shot pitch and yaw, and Figs 6 and 7 show the shot bounce velocities. It may be noted that Figs 6 and 7 also show the result of the flexible shot with the modulus of elasticity increased by a factor 100 , but this overlays the rigid shot result as described above. It may also be noted that a straight barrel was used in the simulation. The important point shown in these figures is that the results of the flexible shot (with the correct modulus of elasticity) are very different from the rigid shot results. This shows that the inclusion of the flexibility of the shot may have an important effect on the overall dynamics and launch characteristics.

\section{Conclusions}

Spin has successfully been incorporated into the simulation of a flexible shot. The results show that the model can simulate shaft whirl, and that the gyroscopic forces, pressure forces and band forces are simulated correctly.

Comparisons between a spinning rigid shot model and the spinning flexible shot model show that the flexibility can have a significant effect on the overall dynamics of the shot, and hence the launch characteristics.

\section{References}

[1] D.N. Bulman, Simulation of Gun Dynamics to Improve Accuracy and Consistency, 15th International Symposium on Ballistics, Israel, 21-24 May 1995.

[2] SIMBAD (Simulation of Barrel Dynamics), User Manual, Danby Engineering Ltd, High Gill House, Low Row, Richmond, N. Yorks, DL11 6NE, UK. Tel/Fax +44 (0) 1748 886345/886014, June 1998

[3] SIMVED (Simulation of Vehicle Dynamics), User Manual, Danby Engineering Ltd, High Gill House, Low Row, Richmond, N. Yorks, DL11 6NE, UK. Tel/Fax +44 (0) 1748 886345/886014, July 1998.

[4] T.F. Erline, Dynamic Analysis of a Puller Sabot Concept, 7th US Symposium on Gun Dynamics, Newport, Rhode Island, 11-13 May 1993.

[5] D.N. Bulman, The In-bore Bending Dynamics of Long Rod Projectiles, 13th International Symposium on Ballistics, Stockholm, 1-3 June 1992.

[6] S. Wilkerson, D.A. Hopkins and B. Held, Techniques for Modeling Bullet Exit Conditions Predicted by Transient Finite Element Models, 8th US Symposium on Gun Dynamics, Newport, Rhode Island, 14-16 May 1996.

[7] D.A. Rabern and K.A. Bannester, Finite Elements Models to Predict the Structural Response of $120 \mathrm{~mm}$ Sabot/Rods During Launch, 6th US Army Symposium on Gun Dynamics, Tamiment, Pennsylvania, 15-17 May 1990.

[8] D.N. Bulman, A comparison of theoretical jump for rifled and non rifled barrels, 5th US Army Symposium on Gun Dynamics, Rensselaerville, NY, 23-25 Sep. 1987.

[9] D.N. Bulman and J.B. Hoyle, An experimental Validation of the SIMBAD Gun Dynamics simulation Package for Flexble Saboted Long Rod Projectiles, 7th US Symposium on Gun Dynamics, Newport, Rhode Island, 11-13 May 1993.

[10] G. Barker, An Experimental Investigation into In-bore Yaw in a Rifled Barrel using the RMCS Air Powered Gun, 5th US Army Symposium on Gun Dynamics, Rensselaerville, NY, 23-25 September 1987.

[11] P.W. Fuller, Measurement of Yaw In-bore, 5th International Symposium on Ballistics, Toulouse, 1980. 

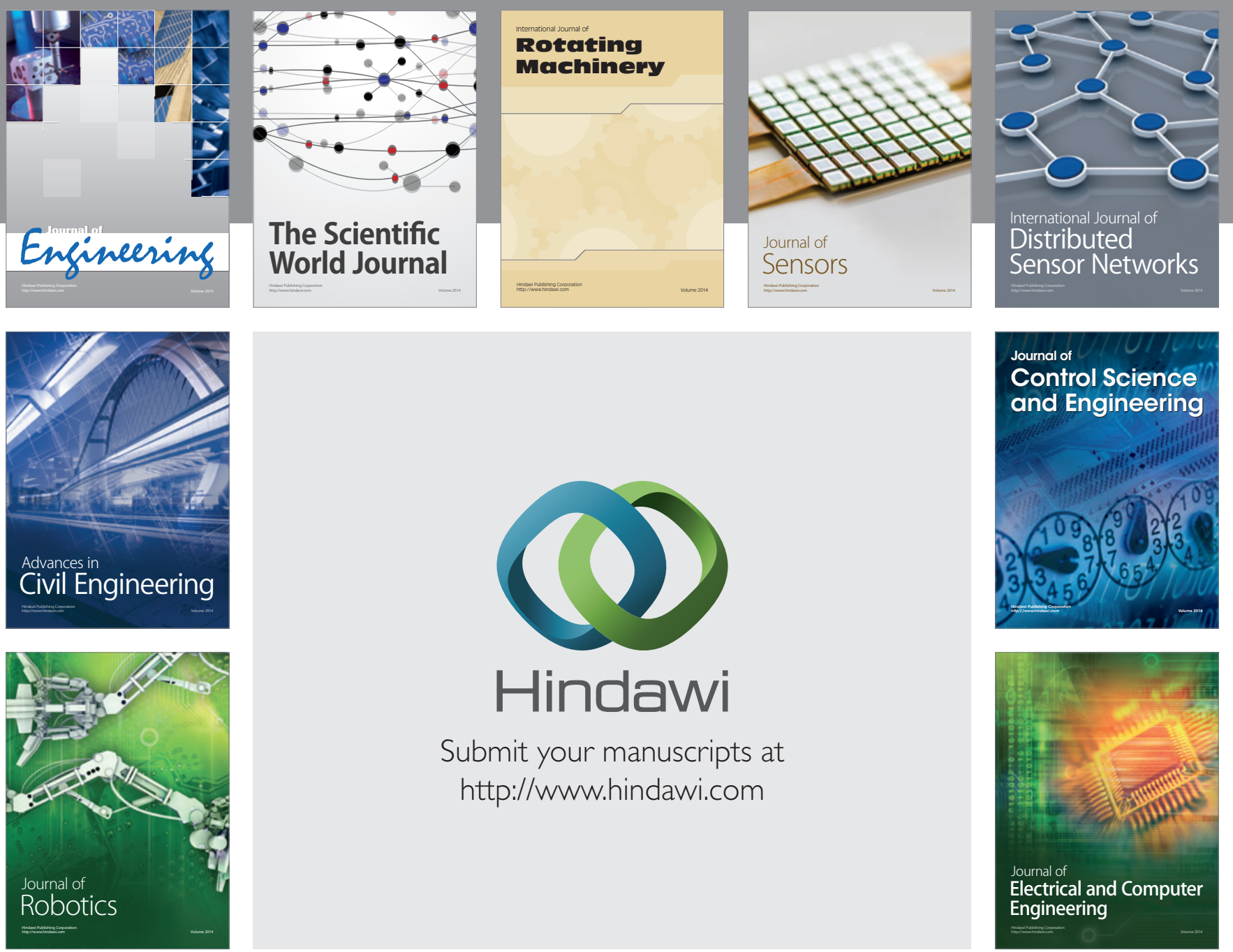

Submit your manuscripts at

http://www.hindawi.com
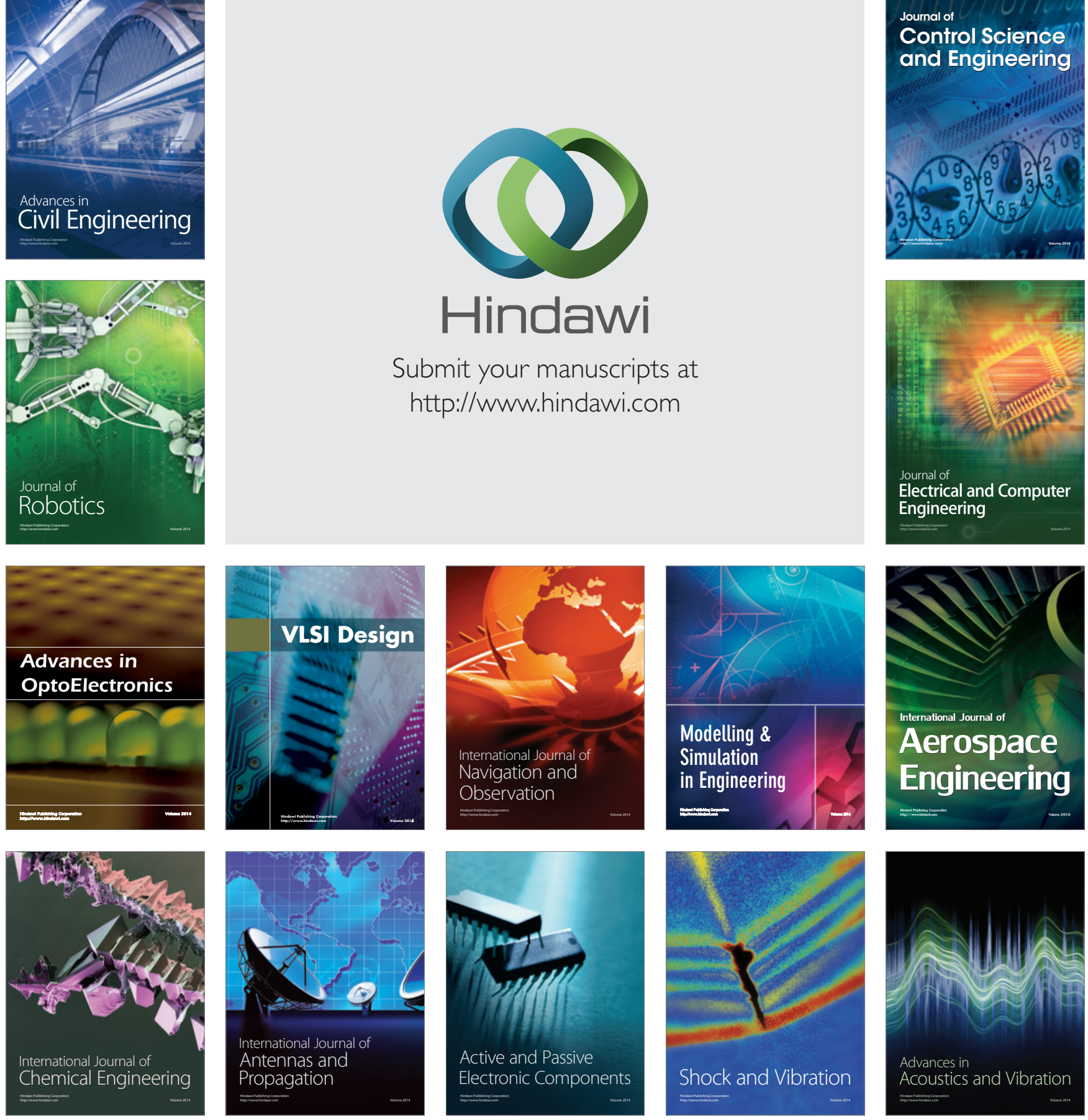